\title{
Current-voltage scaling of a Josephson-junction array at irrational frustration
}

\author{
Enzo Granato \\ Condensed Matter Physics Group, International Centre for Theoretical Physics, 34100 Trieste, Italy \\ and Laboratório Associado de Sensores e Materiais, Instituto Nacional de Pesquisas Espaciais, \\ 12225 - São José dos Campos, São Paulo, Brazil*
}

(Received 9 July 1996)

\begin{abstract}
Numerical simulations of the current-voltage characteristics of an ordered two-dimensional Josephsonjunction array at an irrational flux quantum per plaquette are presented. The results are consistent with a scaling analysis that assumes a zero-temperature vortex-glass transition. The thermal-correlation length exponent characterizing this transition is found to be significantly different from the corresponding value for vortex-glass models in disordered two-dimensional superconductors. This leads to a current scale where nonlinearities appear in the current-voltage characteristics decreasing with temperature $T$ roughly as $T^{2}$ in contrast with the $T^{3}$ behavior expected for disordered models. [S0163-1829(96)51538-9]
\end{abstract}

There has been an increasing interest in two-dimensional Josephson-junction arrays as a model system to study disorder and frustration effects as found in high- $T_{c}$ superconductors and spin-glass systems. ${ }^{1}$ In Josephson-junction arrays, frustration without disorder can in principle be introduced by applying an external magnetic field on a perfect periodic array. The frustration parameter $f$, the number of flux quantum per plaquette, is given by $f=\phi / \phi_{o}$, the ratio of the magnetic flux through a plaquette $\phi$ to the superconducting flux quantum $\phi_{o}=h c / 2 e$, and can be tuned by varying the strength of the external field. Frustration can be viewed as resulting from a competition between the underlying periodic pinning potential of the array and the periodicity of the vortex lattice. ${ }^{2}$ At a rational value of $f$, the ground state is a commensurate pinned vortex lattice leading to discrete symmetries in addition to the continuous $U(1)$ symmetry of the superconducting order parameter. In particular, for $f=1 / 2$ which has been intensively studied both experimentally and theoretically, a superconducting phase transition takes place at finite temperatures with an interplay of $U(1)$ and discrete $Z_{2}$ symmetry. ${ }^{3}$ At irrational values of $f$, the behavior is much less understood since the vortex lattice is now incommensurate with the array. The ground state consists of a disordered vortex pattern ${ }^{4}$ lacking long-range order which can also be regarded as a vortex-glass state without disorder. One cannot completely rule out a possible glass transition at finite temperatures as has been suggested by Halsey ${ }^{4}$ but other arguments suggest a zero-temperature transition. ${ }^{2,5}$ In any case, on the basis of a close analogy between this system and gauge glass models of disordered superconductors, one expects metastable states with long relaxation times and a nonzero critical current at zero temperature. ${ }^{4,5}$ In fact, measurements of $I-V$ characteristics in two-dimensional superconducting wire networks at an irrational frustration ${ }^{6}$ have been interpreted within the scaling analysis of a vortex glass transition as in three-dimensional disordered superconductors where there is evidence of a finite-temperature glass transition. ${ }^{7}$ In the case of superconducting wires, the behavior is likely to be dominated by a mean field transition which should correspond to the behavior at higher dimensions. This could provide a possible explanation for the observed scaling behavior of a finite-temperature transition. On the other hand, no evidence was found in experiments on twodimensional proximity-coupled Josephson-junction arrays ${ }^{8}$ where phase fluctuations are expected to be more important. In disordered superconductors, studies of different models of the vortex glass tend to agree that in two dimensions a vortex-glass transition takes place only at zero temperature ${ }^{7}$ which is supported both by numerical simulations ${ }^{9}$ and experiments. ${ }^{10}$ Although being completely different in the nature of their ground states, the similarity of the behavior of these two systems, specially regarding slow relaxation vortex dynamics and a possible zero-temperature transition in low dimensions, strongly suggests that a two-dimensional array at irrational $f$ should behave as a zero-temperature vortex glass. In this case, the appropriate scaling of the $I-V$ characteristics should be the one corresponding to a zerotemperature transition while the different nature of the ground states should be reflected in the value of the critical exponents as a different universality class. It seems therefore worthwhile to investigate to which extent an array at irrational $f$ can be described as a zero-temperature vortex glass.

In this work, we present simulations of the currentvoltage characteristics of a Josephson-junction array at an irrational flux quantum per plaquette $f=(3-\sqrt{5}) / 2$, a golden irrational, and an scaling analysis ${ }^{7}$ which assumes a zerotemperature vortex-glass transition. The results are consistent with the scaling assumption and allow for an estimation of the thermal-correlation-length critical exponent $\nu$ characterizing the zero-temperature transition. This critical exponent is found to be significantly different from the corresponding value for vortex-glass models in disordered two-dimensional superconductors. As a result, the current density scale, $J_{n l} \sim T^{1+\nu}$, where nonlinearities appear in the current-voltage characteristics decreases with temperature roughly as $T^{2}$ in contrast with the $T^{3}$ behavior expected for disordered models. ${ }^{9}$ This could provide a signature of the behavior at irrational frustration in experimental studies of ordered arrays of Josephson junctions.

We considered an ordered array of Josephson junctions defined on a square lattice. The $I-V$ characteristics of the array were computed using an overdamped Langevin mo- 


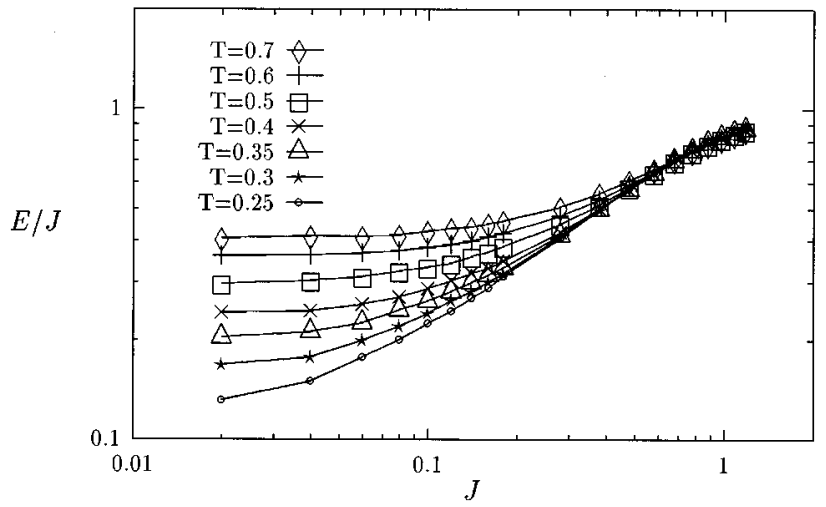

FIG. 1. Nonlinear current-voltage characteristics at different temperatures for a system size $L=34$.

lecular dynamics as described by Falo et al. ${ }^{11}$ One allows for a capacitance to the ground $C_{o}$ in addition to a shunt resistance $R_{0}$ between superconducting grains. In the overdamped limit, this particular dynamics reduces to the standard resistance shunt junction (RSJ) model ${ }^{12}$ commonly used in dynamical simulations. ${ }^{13}$ The Langevin equations can be written as

$$
\begin{aligned}
C_{o} \frac{d^{2} \theta_{i}}{d t^{2}}+\frac{1}{R_{o}} \sum_{j} \frac{d\left(\theta_{i}-\theta_{j}\right)}{d t} \\
\quad=-I_{c} \sum_{j} \sin \left(\theta_{i}-\theta_{j}-A_{i j}\right)+I_{i}^{\mathrm{ext}}+\sum_{j} \eta_{i j},
\end{aligned}
$$

where $\eta_{i j}$ is a Gaussian white noise satisfying

$$
\begin{gathered}
\left\langle\eta_{i j}(t)\right\rangle=0, \\
\left\langle\eta_{i j}(t) \eta_{k l}\left(t^{\prime}\right)\right\rangle=\frac{2 k_{B} T}{R_{o}} \delta_{i j, k l} \delta\left(t-t^{\prime}\right)
\end{gathered}
$$

and $i, j$ are nearest-neighbor pairs. $I^{\text {ext }}$ is the external current and $I_{c}$ the Josephson-junction critical current. The bond variables $A_{i j}$ are constrained to $\Sigma_{i j}=2 \pi f$ where $\Sigma_{i j}$ represents a direct sum around a plaquette. We use units where $\hbar / 2 e=1, R_{o}=1, I_{c}=1$ and set the parameter $I_{c} R_{o}^{2} C_{o}=0.5$ in the simulations, corresponding to the overdamped regime. To determine the nonequilibrium voltage across the system, an external current $I$ is injected uniformly with density $J=I / L$ along one edge of a square array of size $L \times L$ and extracted at the opposite one. Periodic boundary conditions are used in the transverse direction to the current. The average voltage drop $V$ across the system is given by

$$
V=\frac{1}{L} \frac{\hbar}{2 e} \sum_{j=1}^{L}\left\langle\frac{d \theta_{1, j}}{d t}-\frac{d \theta_{L, j}}{d t}\right\rangle
$$

and the average electric field by $E=V / L$. The dynamical equations were integrated numerically using typically a time step $\delta t=0.03 \tau\left[\tau=\hbar /\left(2 e R_{o} I_{c}\right)\right]$ and averages computed with $2 \times 10^{5}$ time steps for each calculation. Lattices of sizes $L=21$ and $L=34$ were used in the simulations without significant size dependence.

In Fig. 1, we show the nonlinear $I-V$ characteristics as a

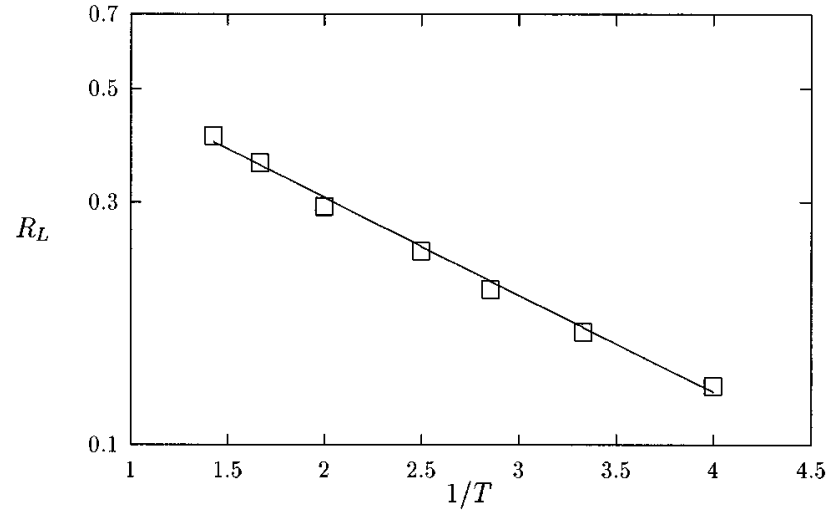

FIG. 2. Temperature dependence of the linear resistance as estimated from $E / J$ at $J=0.02$.

function of temperature. At small current densities $J$, the nonlinear resistivity $E / J$ shows a linear contribution where $E / J$ is a constant. The linear resistivity decreases rapidly with decreasing temperature. For increasing $J$, the resistivity cross over to a nonlinear behavior at a critical current which also decreases with decreasing temperature. For $T<0.25$, the linear behavior presumably occurs at current densities much smaller than the lowest value used in the simulations, $J=0.02$. However, in order to confirm this behavior better statistics and long equilibration times would be required to obtain reliable data. This is consistent with a kind of frozen state for temperatures below $T \sim 0.25$ as indicated in Monte Carlo simulations ${ }^{4}$ where a nonzero Edwards-Anderson order parameter $q(t)=\left\langle\vec{S}_{i}\right\rangle^{2}$, where $\vec{S}=[\cos (\theta), \sin (\theta)]$, averaged over long times $t$, was observed below this temperature. This implies a diverging relaxation time $\tau \sim \int_{0}^{\infty} q(t) d t$, which prevents one to obtain fully equilibrated data in this lowtemperature regime.

In Fig. 2 we show the behavior of the linear resistance as a function of temperature, estimated from $E / J$ at the smallest current. It appears consistent with an activated Arrhenius behavior with an estimated energy barrier $E_{b} \sim 0.45$. We note that this energy barrier is higher than the single vortex barrier in a periodic array, ${ }^{14} E_{b} \sim 0.19$. This suggests that many vortices are involved in the activation process and the vortex dynamics could be the result of collective motion of correlated vortices within a finite length scale.

The behavior of the nonlinear $I-V$ characteristics described above can be understood within a scaling theory which assumes a second-order phase transition at zero temperature. ${ }^{7}$ This theory has been applied to the study of models of vortex glasses in disordered two-dimensional superconductors ${ }^{9,10}$ but it can be equally applied to our case once a zero-temperature transition is accepted. Basically, the behavior of the $I-V$ curves is strongly affected by the increasing correlation length $\xi$ which is assumed to diverge at zero temperature as $\xi \sim T^{-\nu}$ with a thermal correlation exponent $\nu$. At small current densities $J$, the behavior is dominated by vortex dynamics at length scales larger than $\xi$ where the system behaves as a vortex liquid with a linear resistance $R_{L}$. At higher currents, one probes length scales smaller than $\xi$ where the vortex dynamics is expected to be 
nonlinear. Near the transition, the nonlinear $I-V$ characteristics can be cast into a scaling form ${ }^{7,9}$

$$
\frac{E}{J R_{L}}=g\left(\frac{J}{T^{\nu+1}}\right),
$$

where $g$ is a scaling function which has the property that $g(x) \rightarrow 1$ when $x \rightarrow 0$. Since at nonzero temperatures the correlation length is finite, vortex motion will proceed by thermal activation leading to a linear resistance in the form

$$
R_{L} \propto \exp \left(-E_{b} / T\right),
$$

where $E_{b}$ is a typical barrier height for vortex motion within a correlation length $\xi$. In general, the barrier energy should also scale with $\xi$ as $E_{b} \sim \xi^{\psi}$ with a new exponent $\psi \geqslant 0$ but the present data do not allow a clear identification of this behavior. The Arrhenius plot in Fig. 2 is consistent with the simplest scenario $\psi=0$ with $E_{b} \sim 0.45$. The characteristic time scale $\tau$ should also have an exponential behavior $\tau \sim \exp \left(E_{b} / T\right)$ leading to very slow relaxation at low temperatures consistent with the results above and Monte Carlo simulations. ${ }^{4}$ The estimated $E_{b}$ being larger than the single vortex pinning potential ${ }^{14} E_{p}=0.19$ is also consistent with a relaxation process involving collective rearrangement of pinned vortices. From the scaling behavior above, one also sees that the characteristic current density at which nonlinear behavior is expected to set in varies as $J_{n l} \sim T^{1+\nu}$. In a finite system, there will also be a dependence on the system size but the system sizes we have used, $L=21$ and $L=34$, do not show significant corrections.

In Fig. 3, we show a scaling plot of the $I-V$ characteristics data of Fig. 1. From the above scaling analysis all data should collapse on to the same curve if $\nu$ is chosen correctly, assuming size corrections are small. Choosing different values of $\nu$, we find that a reasonable scaling behavior is obtained for $\nu \sim 0.9 \pm 0.2$. This value of $\nu$ should be compared with the corresponding value for the gauge glass model ${ }^{9}$ of disordered superconductors in a magnetic field, $\nu \sim 2$. As

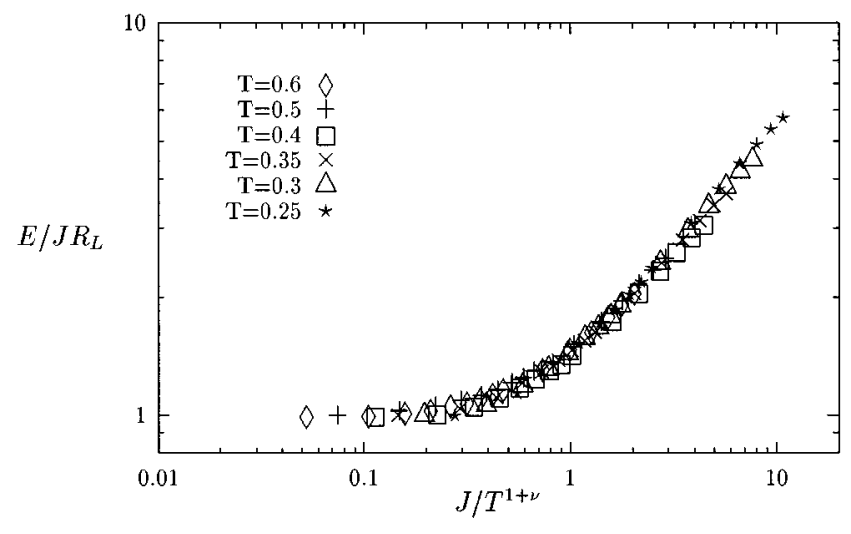

FIG. 3. Scaling plot of the nonlinear current-voltage characteristics of Fig. 1 for $\nu=0.9$.

expected, the different nature of the two vortex-glass transitions is reflected in the values of this critical exponent. The sharp difference between the critical exponents also leads to a completely different behavior for the crossover current density which is expected to behave as $J_{n l} \sim T^{2}$ in contrast with the $\sim T^{3}$ behavior for models of vortex glasses in disordered superconductors. In the experimental systems additional complications may arise since a higher-order rational value can always be found within the confidence interval of a measured value of $f$.

In conclusion, the current-voltage characteristics of an ordered Josephson-junction array at an irrational flux quantum per plaquette were studied using a scaling analysis of a zerotemperature transition. The correlation critical exponent is found to be significantly different from the corresponding value for vortex-glass models in disordered two-dimensional superconductors. This leads to a current scale where nonlinearities sets in decreasing with temperature roughly as $T^{2}$ in contrast with the $T^{3}$ behavior expected for disordered models. It is suggested that this behavior could provide an experimental signature for an array at irrational frustration.
* Permanent address.

${ }^{1}$ See articles in Proceedings of Workshop on Josephson Junction Arrays, 1995 [Physica B 222, 1 (1996)].

${ }^{2}$ S. Teitel and C. Jayaprakash, Phys. Rev. Lett. 51, 1999 (1983)

${ }^{3}$ E. Granato, J.M. Kosterlitz, and M.P. Nightingale, Physica B 222, 266 (1996), and references therein.

${ }^{4}$ T. Halsey, Phys. Rev. Lett. 55, 1018 (1985).

${ }^{5}$ M. Y. Choi and S. Doniach, Phys. Rev. B 31, 4516 (1985).

${ }^{6}$ F. Yu, N. E. Israeloff, A. M. Goldman, and R. Rojko, Phys. Rev. Lett. 16, 2535 (1992).

${ }^{7}$ D. S. Fisher, M. P. A. Fisher, and D. A. Huse, Phys. Rev. B 43, 130 (1991).
${ }^{8}$ J. P. Carini, Phys. Rev. B 38, 63 (1988).

${ }^{9}$ R. A. Hyman, M. Wallin, M. P. A. Fisher, S. M. Girvin, and A. P. Young, Phys. Rev. B 51, 15304 (1995).

${ }^{10}$ C. Dekker, P. J. M. Wóltgens, R. H. Koch, R. W. Hussey, and A. Gupta, Phys. Rev. Lett. 69, 2717 (1992).

${ }^{11}$ F. Falo, A. R. Bishop, and P. S. Lomdahl, Phys. Rev. B 41, 10983 (1990).

${ }^{12}$ S. R. Shenoy, J. Phys. C 18, 5163 (1985).

${ }^{13}$ J. S. Chung, K. H. Lee, and D. Stroud, Phys. Rev. B 40, 650 (1989).

${ }^{14}$ C. J. Lobb, D. W. Abraham, and M. Tinkham, Phys. Rev. B 27, 150 (1993). 